\title{
Depinning of elastic manifolds
}

\author{
Alberto Rosso, Alexander K. Hartmann*, Werner Krauth \\ CNRS-Laboratoire de Physique Statistique \\ Ecole Normale Supérieure, \\ 24, rue Lhomond, 75231 Paris Cedex 05, France
}

\begin{abstract}
We compute roughness exponents of elastic $d$-dimensional manifolds in $(d+1)$-dimensional embedding spaces at the depinning transition for $d=1, \ldots, 4$. Our numerical method is rigorously based on a Hamiltonian formulation; it allows to determine the critical manifold in finite samples for an arbitrary convex elastic energy. For a harmonic elastic energy $\left(\Delta^{2}\right.$-model), we find values of the roughness exponent between the one-loop and the two-loop functional renormalization group result, in good agreement with earlier cellular automata simulations. We find that the $\Delta^{2}$-model is unstable with respect both to slight stiffening and to weakening of the elastic potential. Anharmonic corrections to the elastic energy allow us to obtain the critical exponents of the quenched KPZ class.
\end{abstract}

Elastic manifolds in random media are an important issue of current research in statistical physics. zero-temperature motion of these manifolds, subject to a driving force $f$, the "depinning threshold" $f_{c}$ plays a central role: For forces $f>f_{c}$, the elastic manifold moves with finite velocity, while it is pinned for $f \leq f_{c}$. Among the subjects studied at and around $f_{c}$ are the "creep" motion for $f<f_{c}$ at finite temperature, the scaling of the velocity for small forces $f \gtrsim f_{c}$, and the statistical properties of the pinned critical manifold at $f_{c}$, especially its roughness exponent $\zeta$.

If one neglects velocity-dependent terms in the equations of motion of the manifold, which one assumes to be a single-valued function $h(\mathbf{x}, t)$ (no overhangs), its dynamics is governed by a functional $E(\{h, \mathbf{x}\})$ incorporating potential energy due to the driving force $f$, the disorder $\eta(\mathbf{x}, h)$, as well as its internal elastic energy $E_{\mathrm{el}}$,

$$
\partial_{t} h(\mathbf{x}, t)=-\frac{\partial E}{\partial h(\mathbf{x})}=f+\eta(\mathbf{x}, h)-\frac{\partial E_{\mathrm{el}}}{\partial h(\mathbf{x})} .
$$

Note that $\mathbf{x}$ is a $d$-dimensional vector, in an embedding space of dimension $d+1$. Dimensional analysis suggests that the harmonic approximation for $E_{\mathrm{el}}$ provides the only relevant term for the interplay between disorder and elasticity. This yields the quenched Edwards-Wilkinson (EW) equation

$$
\partial_{t} h(\mathbf{x}, t)=f+\eta(\mathbf{x}, h)+a \nabla^{2} h(\mathbf{x}) .
$$

The model described by eq. (2) has been an important testing ground for the concepts and techniques originally developed in the field of critical phenomena. Functional renormalization group techniques 3 were used to perturbatively compute critical exponents of the quenched EW equation, which were believed to describe generic driven manifolds at the depinning threshold.

According to the framework provided by the functional renormalization group, the manifold is flat $(\zeta=0)$ for

\footnotetext{
*present address: Institut für Theoretische Physik, Universität Göttingen, Bunsenstr. 9, 37073 Göttingen, Germany
}

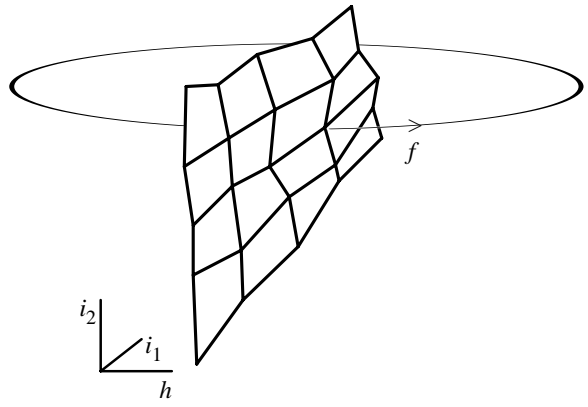

FIG. 1: Schematic description of a $d=2-$ dimensional manifold, driven by a force density $f$. The $(d+1)$-dimensional embedding space is periodic in the manifold's dimensions $i_{1}, \ldots, i_{d}$, but also in the variable $h$.

$d \geq d_{\mathrm{uc}}=4$. Below this upper critical dimension, the roughness exponent is expressed in an fexpansion in $\epsilon=$ $4-d$. The first order (one-loop) term 3 - of the expansiop gives $\zeta=\epsilon / 3$. Initially, this result was pelieved exact $t$ for all $d=1,2,3$. However, Chauve et al . $^{3}$ have obtained the two-loop corrections, and found them to be non-zero.

In this work, we compute the roughness exponent of critical manifolds in finite $(d+1)$-dimensional samples for $d=1, \ldots, 4$. Our approach is rigorously based on a Hamiltonian formulation, as we study directly the dynamics of eq. (11) for a general convex elastic energy $E_{\mathrm{el}}$ : A powerful numerical algorithm allows to solve this problem for a slightly modified version of eq. (1), with a discretized vector $\mathbf{x}(\mathbf{x} \rightarrow \mathbf{i})$ and a continuous variable $h$. The short-range elastic energy depends on the nextneighbor distances $\Delta_{\mathbf{i}, \delta}$ between lattice point $\mathbf{i}$ and its $2 d$ neighbors $\mathbf{i} \pm \boldsymbol{\delta}_{j}$, as shown in Fig. i:

$$
\frac{1}{2} \sum_{j=1}^{d} \sum_{ \pm \boldsymbol{\delta}_{j}} E_{\mathrm{el}}\left(\left|h_{\mathbf{i}}-h_{\mathbf{i}+\boldsymbol{\delta}_{j}}\right|\right) .
$$

We introduce periodic boundary conditions for $\mathbf{x}$ and $h$ (cf Fig. 1) 1 Continuous, periodic random potentials are constructed from $M$ normally distributed random variables. 8 In such a system, the sample-dependent critical force $f_{c}$ is well defined: pinned configuration with 
$v_{\mathbf{i}}=0 \forall \mathbf{i}$ do (do not) exist far driving forces smaller (larger) than $f_{c}$. Our algorithm $\$$ allows to decide quickly whether pinned configurations exist at a given driving force, and then zooms in on the critical force and the critical manifold.

For the first time, we are thus able to unambiguously compute the object of most direct theoretical interest in this problem, namely the critical manifold, in dimensions $d=2,3,4$. We computd 9 the roughness exponent $\zeta$ from the disorder-averaged mean square deviations of the critical manifolds $h^{c}$ :

$$
W^{2}(L)=\overline{\left\langle\left(h^{c}-\left\langle h^{c}\right\rangle\right)^{2}\right\rangle} \sim L^{2 \zeta} \text { for } L \rightarrow \infty .
$$

As the width of the manifold is of order $\sim L^{\zeta}$, we scale the lateral extension of the sample at least as $M \sim L^{\zeta}$.

Especially in higher dimensions, direct simulations of eq. (2) are not viaple, because they violate a crucial nopassing theorem 10 which we respect.

Because of the expected universality and the difficulties of direct simulation, attempts to compute the critical exponents of the quenched EW equation considered discrete systems with non-hamiltonian dynamic rules. These cellular automata model $\$ 11.12$ are not clearly connected with an equation of motion in the continuum limit 13 and have often given only rough estimates for $\zeta$.

Nevertheless, simulations using cellular automata illustrated that critical elastic manifolds in random media are not generally described by the quenched EW equation but may fall into two broad classes: The automatal, 4 summarized in Ref. 15, yield critical exponents which are close to those obtained by analytical work on eq. (2); on the other hand the automata 11.22 summarized in Ref. 16 give very different values for the exponents, incompatible with the results obtained by rengrmalization group results, but closer to experiment 12,17, 28 There has been much confusion about the actual values of these exponents for $d>1$. It is not clear whether the upper critical dimension of this class is also $d_{\mathrm{uc}}=4$.

In our controlled Hamiltonian approach, we recover this very rich behavior as a dependence of the roughness exponent $\zeta$ on the functional form of the elastic energy: We investigate elastic energies of the form $E_{\text {el }} \propto|\Delta|^{\gamma}$, and refer to them as $\Delta^{\gamma}$-models. The $\Delta^{2}$-model studies directly the eq. (2). Models with $\gamma \neq 2$ differ in their critical behavior from the harmonic model. A special role is played by the $\Delta^{4}$-model, which represents the first non-harmonic corrections of a general elastic energy $E_{\mathrm{el}} \sim a \Delta^{2} / 2+b \Delta^{4} / 12+\ldots$

We first discuss this important issue for an elastic string $(d=1)$ in a 2 -dimensional medium, which has been abundantly studied in the past. As shown in Fig. 2, we find for the $\Delta^{2}$-model a value of the roughness exponent $\zeta_{\Delta^{2}}=1.26 \pm 0.01$, which compares well with the two-loop calculation of Chauve et al., while clearly excluding the one-loop result $\zeta_{\Delta^{2}}^{\text {oneloop }}=1$. A value in excess of one for the $(1+1)$-dimensional roughness has been found in direct numerical integrations of the equa-

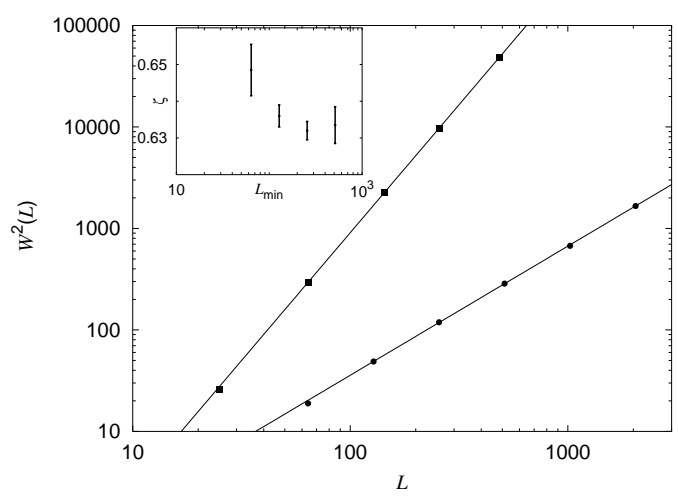

FIG. 2: Mean square extension $W^{2}$ vs system size for the $(1+1)$-dimensional elastic string. Upper curve: harmonic elastic energy $E_{\mathrm{el}}(\Delta)=\Delta^{2} / 2 ; M \sim L^{1.5}$. Lower curve: elastic energy $E_{\mathrm{el}}(\Delta)=\Delta^{4} / 4 ; M=L$. The inset shows the estimated value of $\zeta$ for all the data with $L>L_{\min }$ as a function of $L_{\text {min }}\left(\Delta^{4}\right.$-model $)$. The absence of systematic trends leads us to conclude that $\zeta_{\Delta^{2}}=1.26 \pm 0.01, \zeta_{\Delta^{4}}=0.635 \pm 0.005$.

tions of motion 19 in Monte Carlo simulations 120 and in the cellular automata of Ref. 15.

Figure 2 also shows our results for the $\Delta^{4}$-model. We find $21 \zeta_{\Delta^{4}}=0.635 \pm 0.005$. This exponent coincides with the value found in Ref. 16. In lattice models, we already showed that the roughness exponent does not change for the $\Delta^{6}$-model and even in the presence of a metric constraint (bounded $|\Delta|$ ). In this sense, the $\Delta^{4}$-model is only one representative of systems with a stronger than harmonic elastic energy. The values obtained from Fig. 2 are much more precise than the earlier ones.

In this problem, the study of rotational invariance of the equations of motion has occupied a crucial role. In fact, eq. (2), and therefore the equation of motion of our $\Delta^{2}$-model, is invariant under a tilt of the manifold

$$
h(\mathbf{x}, t) \rightarrow h(\mathbf{x}, t)+\sum m_{i} x_{i} .
$$

It was observed 22 that the automata of Ref. 16 present strong dependence of the velocity and of the value of $f_{c}$ on the parameters $m_{i}$. In our $\Delta^{4}$-model, rotational invariance is manifestly broken as an elastic energy $b \Delta^{4} / 12$ generates a piece

$$
b \nabla^{2} h(\nabla h)^{2}
$$

in the equation of motion. From dimensional analysis, the 3 among the orientation-dependent differential operators, the KPZ term $\sim(\nabla h)^{2}$ is more relevant than the term eq. (6). The non-linear KPZ term appears in domaingrowth models without disorder, where it is coupled to the velocity 24 Adding it to eq. (2) constitutes the quenched KPZ equation. Its critical exponents have not been computed within the functional renormalization group, and it is not even clear how this term is generated in the absence of a finite velocity, at depinning.t. 


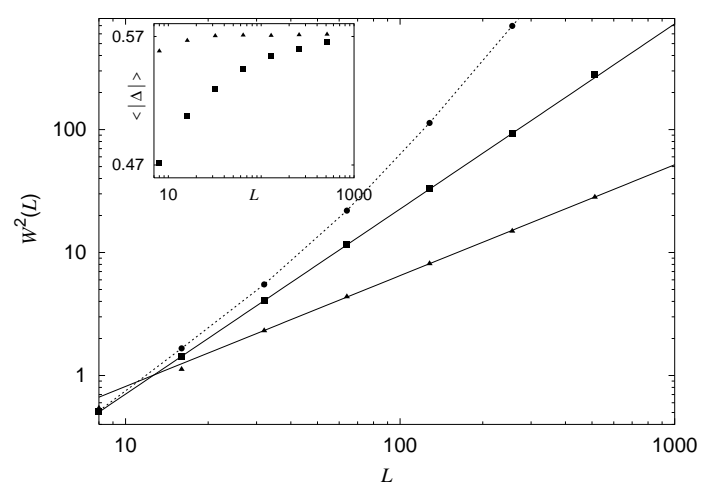

FIG. 3: Mean square extension $W^{2}$ vs system size for the $(2+1)$-dimensional elastic manifold for energies $E_{\mathrm{el}}(\Delta)=$ $\frac{1}{\gamma} \Delta^{\gamma}$ for $\gamma=1.8$ (upper, circles), $\gamma=2$ (middle, squares) and $\gamma=4$ anharmonic energy (lower, triangles); $M=L$. The inset shows the mean local elongation $\langle|\Delta|\rangle$ for $\gamma=2$ and $\gamma=4$. For $\gamma=1.8,\langle|\Delta|\rangle$ clearly diverges (not shown). Error bars are smaller than the symbol sizes.

Kardar et al. 123 have suggested the force anisotropy due to the disorder as a possible generating mechanism, but none of the automata models present such an anisotropy. However, in their construction rules, a metric constraint is hidden. This implements a strong elastic potential, and naturally generates terms like eq. (6). In our opinion, the main unresolved theoretical issue is to understand how this term reduces to the KPZ term under coarse-graining.

As discussed in Ref. 9, there is an easy way to understand why the $(1+1)$-dimensional $\Delta^{2}$-model is unstable to higher-order corrections: A roughness $\zeta>1$ implie 25 that the mean local elongation $\langle|\Delta|\rangle$ grows with the system size at least as $L^{\zeta-1}$, and thus diverges in the thermodynamic limit $L, M \rightarrow \infty$. Higher-order terms in the elastic energy are thus trivially relevant in one dimension. 9

In $2+1$ dimensions, we have studied samples up to size $L^{2} \times M=512^{2} \times 512$. For the $\Delta^{2}$-model (shown in Fig. 3), we find excellent scaling for a roughness $\zeta_{\Delta^{2}}=0.753 \pm 0.002$, which again falls between the results of the one-loop and two-loop renormalization group calculations. Our result improves by an order of magnitude the precision of the previous estimate 15 Note that our algorithm allows us to know, without invoking universality arguments, that the exponent $\zeta_{\Delta^{2}} \sim 0.753$ is that of the two-dimensional quenched EW model.

In Fig. 3, we also show our results for the $\Delta^{4}$-model. We find a different value for $\zeta$, namely $\zeta_{\Delta^{4}}=0.45 \pm$ 0.01 . This last value is significantly smaller than $\zeta \sim 0.48$ found in Ref. 16 using a cellular automaton.

The two-dimensional $\Delta^{2}$-model is not only unstable with respect to stronger elastic potentials, but also to any weaker ones. We have studied the $\Delta^{\gamma}$-model, for $\gamma$ slightly below 2: Already for $\gamma=1.8$ ( $c f$ Fig. 3), we find that the roughness exponent changes drastically, as we

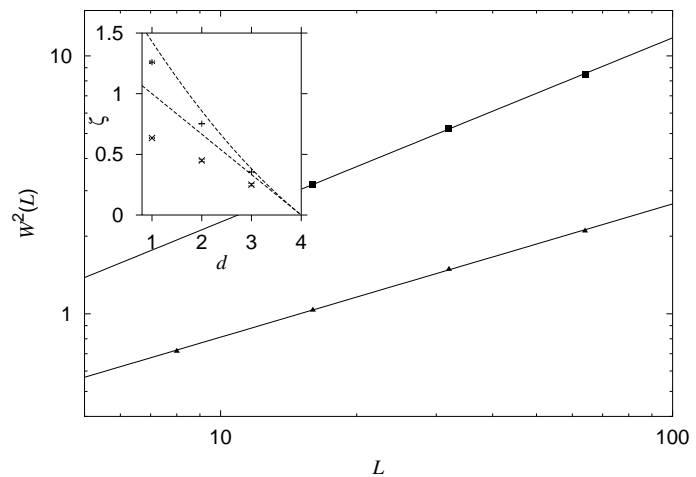

FIG. 4: Mean square extension $W^{2}$ vs system size $(M=$ $L)$ for the $(3+1)$-dimensional elastic manifold for energies $E_{\text {el }}(\Delta)=\frac{1}{4 \gamma} \Delta^{\gamma}$ for $\gamma=2$ (harmonic energy, upper curve) and $\gamma=4$ (lower curve). The inset shows $\zeta_{\Delta^{2}}$ and $\zeta_{\Delta^{4}}$ vs $d$. The lines are the one-loop and two-loop results for the $\Delta^{2}$-model.

obtain clear indications that $\zeta_{\Delta^{1.8}}>1$. This implies that the surface breaks.

We also studied elastic energies $\sim \sqrt{1+\Delta^{2}}$ in the $(2+1)$-dimensional problem. In one dimension, this functional form corresponds to the length of the string, and the $\Delta^{2}$-model is often understood as the first term from an expansion of the length in powers of $\Delta$. The square root is softer than its first expansion coefficient; we again find values of $\zeta_{\sqrt{1+\Delta^{2}}}$ in excess of one for large system sizes.

As in $1+1$ dimensions, we also find for the $(2+1)-$ dimensional $\Delta^{2}$-model indications of a (much slower) divergence of the mean local extension, as shown in Fig. 3 . For the $\Delta^{2}$-model, it seems to diverge logarithmically, whereas for the $\Delta^{4}$-model it saturates already for very small systems. Again it appears that only the $\Delta^{4}$-model has a proper thermodynamic limit for the critical manifold.

We also performed extensive computations in $3+1$ and in $4+1$ dimensions, where we have reached sample sizes of $L^{3} \times M=64^{3} \times 64$ and $L^{4} \times M=32^{4} \times 32$, respectively. For the harmonic model in $3+1$ dimensions, we obtain $\zeta_{\Delta^{2}}=0.355 \pm 0.01$ again in good agreement with the numerical results of Ref. 14, while our result $\zeta_{\Delta^{2}}=0.25 \pm 0.02$ for the $\Delta^{4}$-model is in contradiction to Ref. 16 which suggested an exponent $\zeta \sim 0.38$ larger than for the harmonic case. We checked very carefully that our estimate for $\zeta$ is independent of the parameters $a$ and $b$.

At last, erur algorithm is able to investigate $4+1$ dimensions.2 We have computed manifolds of sizes $L=$ $8,16,32(L=M)$. Even though we have only three points, we find for the $\Delta^{2}$-model an inconsistent fit to a functional form $W^{2}(L) \sim L^{2 \zeta}$ for sizes $L$ where it was already good in $3+1$ dimensions. At the upper critical dimension, one would expect logarithmic behavior for $W^{2}(L)$. The most natural explanation of our findings 


\begin{tabular}{|c|c|c|c|c|}
\hline$d$ & \multicolumn{2}{|c|}{ analytic } & $\zeta_{\Delta^{2}}$ & $\zeta_{\Delta^{4}}$ \\
\cline { 2 - 3 } & $\begin{array}{c}\text { one-loop } \\
1\end{array}$ & two-loop & & \\
\hline 2 & $2 / 3$ & 1.44 & $1.26 \pm 0.01$ & $0.635 \pm 0.005$ \\
\hline 3 & $1 / 3$ & 0.86 & $0.753 \pm 0.002$ & $0.45 \pm 0.01$ \\
\hline
\end{tabular}

TABLE I: Roughness exponents as a function of dimension $d$ for the $(d+1)$-dimensional driven manifold problem, both for the $\Delta^{2}$-model (harmonic elastic energy) and the $\Delta^{4}$-model.

in $4+1$ dimensions is that the $\Delta^{2}$-model's upper critical dimension is $d_{\mathrm{uc}}=4$. This will still have to be confirmed for larger sample sizes.

For the $\Delta^{4}$-model, we also do not find a good fit for $\zeta$, but the deviations from the functional form $W^{2}(L) \sim L^{2 \zeta}$ are less striking than for the $\Delta^{2}$-model. It is unclear to us whether the roughness exponent of the $\Delta^{4}$-model can be higher than for the $\Delta^{2}$-model.

In conclusion, we have directly computed roughness exponents for $d$-dimensional manifolds in a $(d+1)$ dimensional embedding space for $d=1, \ldots, 4$. Some of our findings nicely fit into the existing theoretical framework. For example, the upper critical dimension $d_{\mathrm{uc}}=4$, conjectured on the basis of dimensional power counting, is consistent with our data for the $\Delta^{2}$-model.

In the harmonic case, agreement with the two-loop cal- culation is fair, and the exactness of the one-loop result can be excluded ( $c f$ inset of Fig. (4). Our numerical work relies on no additional hypothesis, as we compute welldefined critical manifolds in finite systems. Even for the harmonic case, though, we would like to understand the divergence of the local scale, for which we find evidence even in $d=2$ : slightly sub-harmonic potentials lead to a break-up of the critical surface.

For the first time we have presented a Hamiltonian model which reproduces the results of the quenched KPZ class. The connection between our $\Delta^{\gamma}$-model and the equations of motion in the continuum limit is transparent. The analysis of the $\Delta^{4}$-model gives a very precise estimate for the value of $\zeta$ as a function of dimension $d$. It would be very interesting to understand how the local differential operators, as $\nabla^{2} h(\nabla h)^{2}$, which correspond to elastic Hamiltonians, renormalize into more relevant terms, as the KPZ term.

Acknowledgments: We thank P. Le Doussal for very helpful discussions all along this work, and also acknowledge stimulating discussions with D. S. Fisher and K. J. Wiese. Our computations were performed on clusters of workstations at Ecole Normale Supérieure, the Institut für Theoretische Physik of the Universität Magdeburg (Germany) and at the Paderborn Center for Parallel Computing (Germany). AKH was financially supported by the DFG (Deutsche Forschungsgemeinschaft) under grant Ha 3169/1-1. $\dagger$ Electronic address: h 2 rtmann@theorie.physik.uni-goettingen.de: kauth@lps.ens.fr

1 M. Kardar, Phys. Rep. 301, 85 (1998).

2 A.-L. Barabási, H. E. Stanley, Fractal Concepts in Surface Growth (Cambridge University Press, Cambridge, 1995).

3 T. Nattermann, S. Stepanow, L.-H. Tang, H. Leschhorn, J. Phys. II France 2, 1483 (1992).

${ }^{4}$ O. Narayan, D. S. Fisher, Phys. Rev. B 48, 7030 (1993).

5 P. Chauve, P. Le Doussal, K. J. Wiese, Phys. Rev. Lett. 86, 1785 (2001).

6 The driving force needs a winding term to account for the periodic boundary conditions.

7 many of the technical issues will be published separately; A. Rosso, W. Krauth, in preparation.

8 A. Rosso, W. Krauth, Phys. Rev. E 65, 025101(R) (2002).

9 A. Rosso, W. Krauth, Phys. Rev. Lett. 87, 187002 (2001).

10 A. A. Middleton, Phys. Rev. Lett. 68, 670 (1992). For a complete proof of Middleton's theorem cf C. Baesens, R. S. MacKay, Nonlinearity 11, 949 (1998).

11 K. Sneppen, Phys. Rev. Lett. 69, 3539 (1992), L.-H. Tang, H. Leschhorn, Phys. Rev. A 45, R8309 (1992).

12 S. V. Buldyrev et al., Phys. Rev. A 45, R8313 (1992).

13 However see J. Vannimenus, B. Derrida, J. Stat. Phys. 105, 1 (2001).
14 H. Leschhorn, Physica A 195, 324 (1993).

15 H. Leschhorn, T. Nattermann, S. Stepanow, L.-H. Tang, Annalen der Physik 61 (1997).

16 S. V. Buldyrev, S. Havlin, H. E. Stanley, Physica A 200, 200 (1993).

17 S. V. Buldyrev et al., Physica A 191, 220 (1992).

18 J. Krim, I. Heyvaert, C. van Haesendonck, Y. Bruyhseraede, Phys. Rev. Lett. 80, 849 (1998).

19 H. J. Jensen, J. Phys. A: Math. Gen. 28, 1861 (1995).

20 A. Rosso, W. Krauth, Phys. Rev. B 65, 012202 (2002).

21 The relation $W^{2} \sim L^{2 \zeta}$ holds only in the limit of large $L$. Dependence of the fit on the smallest of the included $L$-values is checked in the inset of Fig. 8, which testifies to the absence of systematic trends for $L_{\min }>16$.

22 L. A. N. Amaral, A.-L. Barabási, H. E. Stanley, Phys. Rev. Lett. 73, 62 (1994).

23 L.-H. Tang, M. Kardar, D. Dhar, Phys. Rev. Lett. 74, 920 (1995).

24 M. Kardar, G. Parisi, Y. C. Zhang, Phys. Rev. Lett. 56, 889 (1986).

25 L.-H. Tang, H. Leschhorn, Phys. Rev. Lett. 70, 2973 (1993).

26 A. Rosso, W. Krauth, J. Vannimenus, in preparation. 